\title{
Mão Reumatóide: revisão de 124 Próteses MCF de Silicone
}

\author{
J. M. Sousa ${ }^{(1)}$, F. Oliveira ${ }^{(1)}$, R. Clatro $^{(1)}$, P. Cardoso $^{(2)}$, \\ M. Trigueiros ${ }^{(3)}$, C. Silva ${ }^{(4)}$ \\ Centro Hospitalar do Porto - Hospital de Santo António \\ (1) INTERNo Da ESPECIALIDADE DE ORTOPEDIA \\ (2) ASSISTENTE HOSPITALAR \\ (3) Assistente Hospitalar EVEntual \\ (4) Assistente Hospitalar Graduado
}

Correspondência:

Dr. João Alexandre de Melo e Sousa

Calçada da Arrábida n ${ }^{\circ} 1$ C31

4150-106 Porto

Teléf: +351919505033

e-mail: joaoamsousa@yahoo.com.br

Objectivos: O tratamento consensual na doença articular avançada da metacarpofalângica é a artroplastia com interposição de prótese. O objectivo deste trabalho foi avaliar o resultado das artroplastias de silicone realizadas em mãos reumatóides, no serviço de Ortopedia do Hospital de Santo António, nos últimos 15 anos.

Material e método: foram revistas 124 próteses de 26 doentes operados nos últimos 15 anos, avaliando-os clínica e radiologicamente, com aplicação de escalas e algoritmos de avaliação de dor, função pré e pós operatória, resultado estético e satisfação global.

Resultados: A maioria dos doentes apresentava artrite reumatóide, atingimento bilateral sendo avaliado ofollow-up entre 18 meses e 13 anos. A indicação cirúrgica predominante foi a dor seguida e acompanhada pelo défice de função. Utilizou-se a prótese de Swanson em $82 \%$ dos doentes, com correcção do desvio cubital associado em $47 \%$. Houve uma recuperação média de 28 graus do arco de mobilidade articular e 4 graus na escala decimal de dor. Registaram-se 10 complicações (seis fracturas e quatro luxações de prótese). A satisfação global dos doentes foi positiva (67\%) com melhoria das actividades da vida diária e deformidade das mãos.

Conclusão: A prótese de silicone demonstrou ser uma boa opção de tratamento para a doença articu-
Aim: The consensual treatment of advanced articular disease of metacarpophalangeal joint is the interposition arthroplasty with prosthesis that has the objective of preserving mobility and improving articular stability.The goal of this revision was to evaluate the result of the arthroplasties with interposition of silicone prosthesis and re-centering of extensors in patients with rheumatoid hands, done in Orthopaedic department of «Hospital Santo António» over the last 15 years.

Material and method: 124 of 26 patients operated over the last 15 years were reviewed. A clinic and radiological pre and post operatory evaluation with scales, pain and function algorithms, aesthetic and global satisfaction was made.

Results: The majority of patients presented with rheumatoid arthritis, with bilateral involvement and a post-surgery follow-up ranging from 18 months to 13 years. The main surgical indication was pain, followed and accompanied by deficit of function. The Swanson prosthesis was used in $82 \%$ of patients, with cubital deviation being surgically corrected in $47 \%$ of cases. There was an average recuperation of $28^{\circ}$ of mobility arch and 4 degrees in decimal scale of pain. As complications, six fractures and 4 luxations of prosthesis were registered. The global satisfaction of patients was positive (67\%) with daily activities and hand deformity improvement. 
lar reumatóide das MCF com excelentes resultados funcionais, estéticos e dor.

Palavras chave: mão reumatóide, articulação $M C F$, prótese de silicone.
Conclusion: Silicon prosthesis has proven to be a good option for the treatment of articular rheumatoid disease of MCP with excellent results of function, aesthetic and pain.

Key words: rheumatoid hand, MCP articulation, silicone prosthesis.

\section{INTRODUÇÃO}

$\mathrm{O}$ tratamento actual da articulação metacarpofalângica (MCF) (e mão reumatóide), deve seguir um plano integrado na progressão local da doença e na globalidade do membro superior e da condição geral do doente ${ }^{1,2}$. A luxação MCF, destruição articular e desequilíbrio muscular condicionam dor e défice funcional sendo os principais factores de morbilidade e constituindo as principais indicações para cirurgia. As artroplastias de interposição com prótese de silicone têm sido o tratamento de escolha nas deformidades sintomáticas e estabelecidas ${ }^{1,2}$. Este tipo de próteses foi introduzido em 1962 por Swanson e têm como objectivo restaurar a mobilidade funcional e estabilidade podendo, em determinadas situações, evitar a progressão da doença ${ }^{1-3}$. A prótese de silicone depende do processo de encapsulamento para estabilizar a articulação ${ }^{(3)}$. A prótese original de Swanson é uma prótese recta, tendo a possibilidade do uso de estruturas metálicas denominadas grommets. Os grommets caíram em desuso pelo facto de não acrescentarem vantagens no resultado final e por aumentarem o tempo cirúrgico, apesar de existirem estudos que os relacionam com diminuição da osteólise e fractura das próteses (sem que isso influenciasse 0 resultado clínico $)^{4}$. Em 1998 foi introduzida uma prótese de silicone Neuflex da Depuy Jonhson ${ }^{\circledR}$ (produzida em Leeds, Reino Unido) que tem como principal alteração em relação à prótese original de Swanson, a passagem de uma prótese recta para uma angulação volar de $30^{\circ} \mathrm{o}$ que diminui a solicitação da força de flexão $0^{5,6}$. Nas situações de desvio cubital significativo e luxação dos tendões extensores deve associar-se a correcção de teci- dos moles (recentragem dos extensores e transferência de m. intrínsecos) ${ }^{1}$. A cirurgia deve ser ponderada e coordenada com as expectativas do cirurgião e do doente ${ }^{1}$.

O objectivo desta revisão foi avaliar os resultados cirúrgicos das próteses de interposição de silicone na articulação MCF realizadas nos últimos 15 anos no serviço de Ortopedia do Hospital Geral de Santo António (período de janeiro de 1993 a dezembro de 2007), no que diz respeito a dor, função, deformidade, actividades da vida diária e satisfação global destes doentes.

\section{MATERIAL E MÉTODO}

Foram avaliados 124 próteses em 26 doentes com mão reumatóide tratados cirurgicamente com prótese de interposição de silicone da articulação MCF, durante 15 anos. Esta avaliação foi retrospectiva.

A técnica cirúrgica realizada foi a seguinte:

- incisão transversal quando estão envolvidas 3 ou 4 articulações MCF

- incisão longintudinal quando envolvidas 1 ou 2 articulações.

- abordagem articular cubital com capsulotomia da MCF

- sinovectomia

- preparação e colocação do implante no metacarpiano e na falange segundo técnica habitual.

Nos casos de desvio cubital dos dedos, luxação dos tendões extensores e contractura dos músculos intrínsecos efectuamos correcção de tecidos moles: 


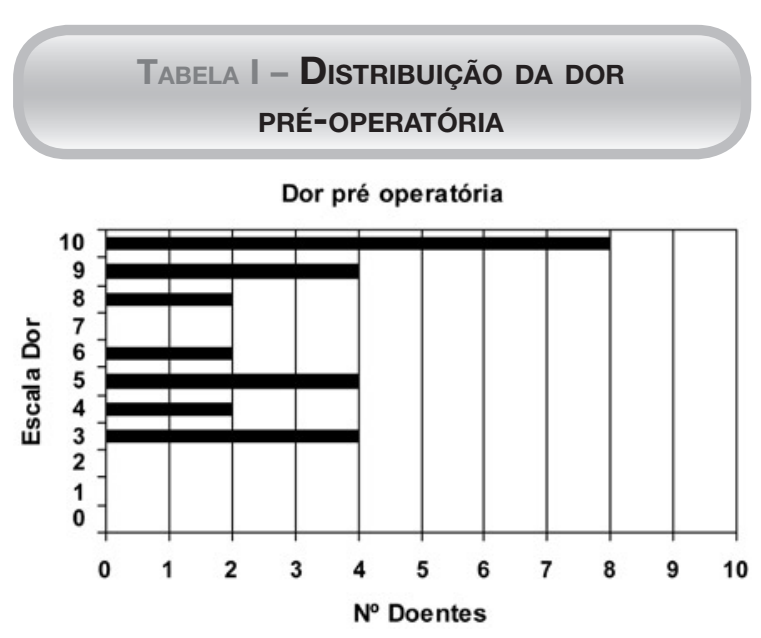

- no desvio cubital dos dedos procedemos à transferência dos tendões dos músculos intrínsecos cubitais para o lado radial do dedo adjacente re-inserindo-o na base da falange.

- na luxação dos extensores procedemos à recentragem dos mesmos pela técnica de Harrison modificada (uma tira de tendão extensor é levantada do lado cubital, passada através da cápsula articular e periósseo da base da falange do lado medial e suturada sobre ela própria)

- na contractura dos músculos intrínsecos fizemos a libertação distal dos mesmos.

Por último é realizada o encerramento da pele com colocação de dreno. A maioria das artroplastias revistas (total de 124) foram próteses de Swanson (102 próteses), seguidas das próteses Neuflex ${ }^{\circledR}$ (22 próteses), tendo sido associada correcção de tecidos moles (recentragem dos tendões extensores e transferência de músculos intrínsecos) em 60 casos. Foram operadas 40 mãos em 26 doentes (14 bilateralmente). Nos casos de atingimento bilateral, a primeira mão a ser submetida a cirurgia foi a mais afectada ou, nos casos simétricos, a mão dominante. Todos os 26 doentes tinham doença reumatológica conhecida, sendo que 20 destes apresentava artrite reumatóide, 3 lúpus eritematoso sistémico e 3 psoríase. Verificou-se uma predominância do sexo feminino (22 casos), um espectro de idades compreendido entre os 32 e 76 anos (média de 57,8 ) e duração da doença de 3 a 46 anos (média de 23,6). O tempo de seguimento pós operatório foi de 18 meses a 13 anos.

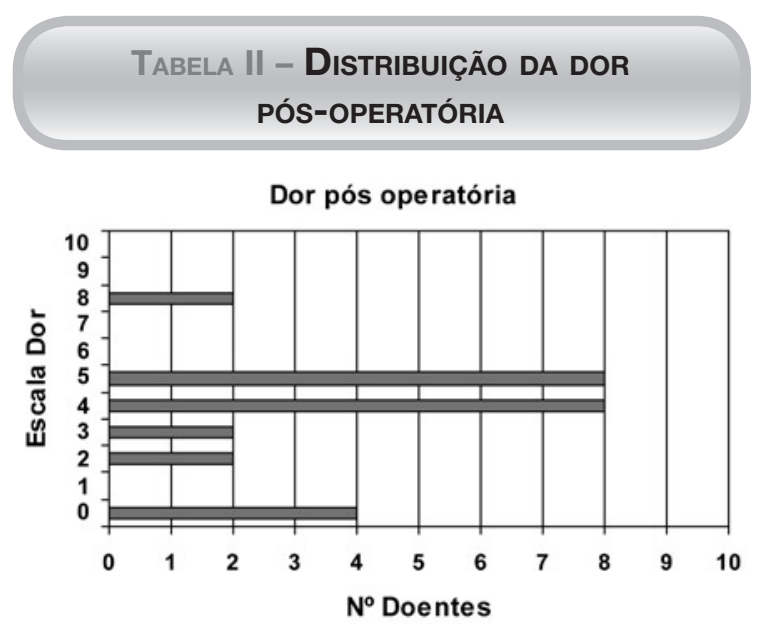

Foi feita avaliação da dor através da escala visual numérica comparando o estado pré e pós-operatório. A avaliação da função foi realizada objectivamente pela melhoria do arco de mobilidade pós-operatório e subjectivamente pela aplicação de um questionário de satisfação das actividades de vida diária após a realização da cirurgia. A melhoria da deformidade e resultado global da cirurgia foi igualmente avaliada através de questionários de satisfação pós-operatórios. Estes questionários aplicados de avaliação subjectiva do doentes foram elaborados pelos autores. Nos questionários de satisfação pós-operatória relativos às actividades da vida diária e melhoria da deformidade foram incluídas as seguintes opções: muito melhor, melhor, igual ou pior. No questionário de satisfação pósoperatória global inclui-se as opções: excelente, muito bom, bom, igual, mau, péssimo. Foram revistos os exames de $\mathrm{Rx}$ e registadas todas as complicações.

Os dados recolhidos foram interpretados de uma forma descritiva, no sentido de perceber a adequação das indicações cirúrgicas e comparando-os com a literatura actual.

\section{RESULTADOS}

A dor pré-operatória apresentava dois subgrupos de doentes com valores a rondar os 9 graus e os 5 graus (Tabela I). Na dor pós operatória verificou-se uma descida para valores entre os 5 e 0 graus (Tabela II). A percentagem de melhoria foi de $82 \%$, com $6 \%$ de casos 


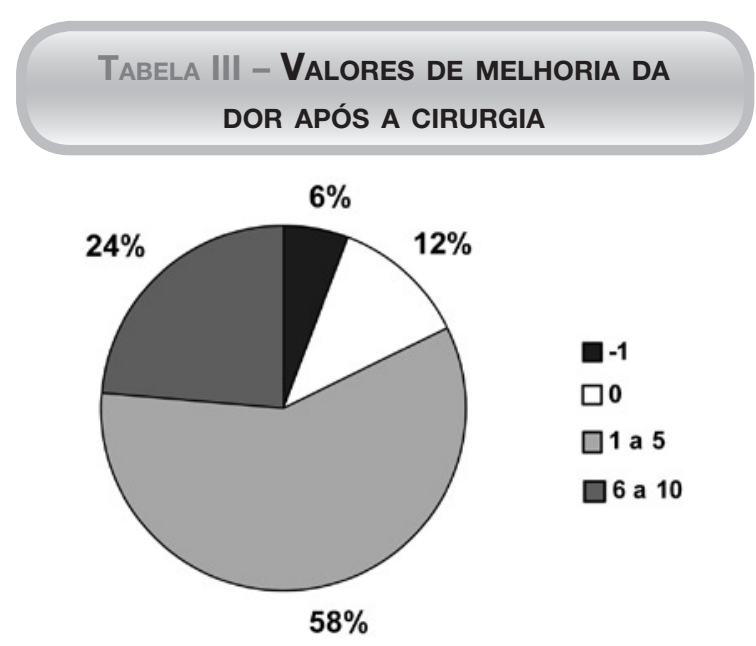

TABela IV - Avaliação funcional: GRAUS DE MELHORIA DO ARCO DE MOBILIDADE

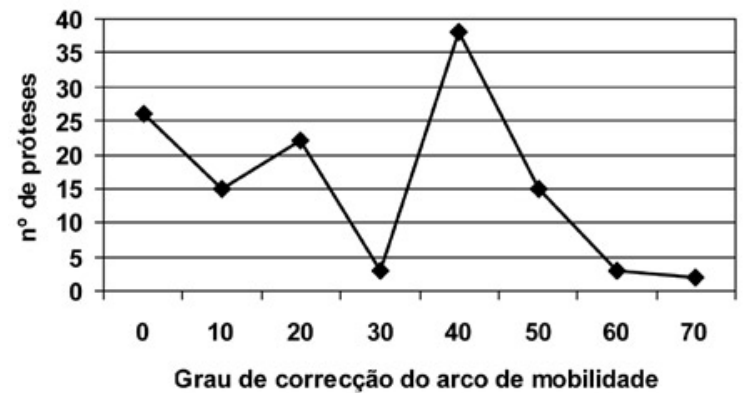

TABela V - Avaliação fUncional ATRAVÉS DE QUESTIONÁRIO DE SATISFAÇÃO PARA AS ACTIVIDADES DA VIDA DIÁRIA

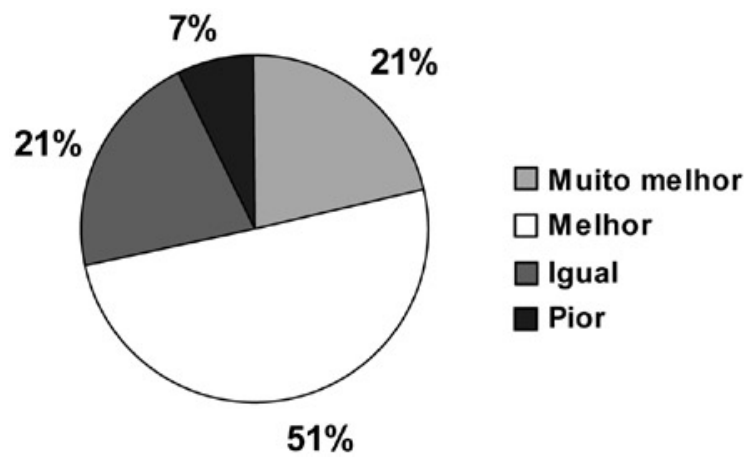

com agravamento e os restantes sem alterações (Tabela III).

Quanto ao resultado funcional, foi verificado objectivamente uma melhoria do arco de mobilidade articular sendo que a maioria dos raios
Tabela VI - Avaliação da deformidade ATRAVÉS DE QUESTIONÁRIO DE SATISFAÇÃO

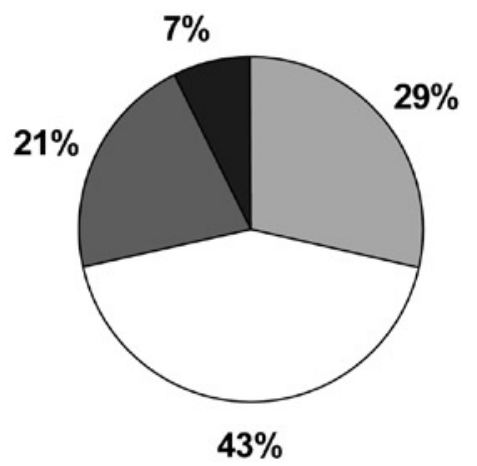

$\square$ Muito melhor $\square$ Melhor $\square$ Igual Pior
Tabela V - Avaliação da satisfação GLOBAL ATRAVÉS DE QUESTIONÁRIO

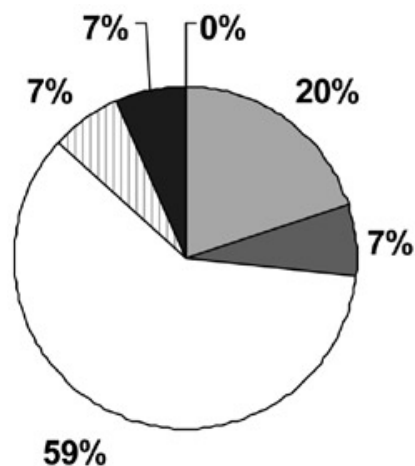

Excelente

Muito Bom

$\square$ Bom

$\square$ Igual

$\square \mathrm{Mau}$

目 Péssimo

$59 \%$

estudados apresentavam valores na ordem dos 40 graus -37 próteses (Tabela IV). No questionário de satisfação das actividades da vida diária registou-se uma melhoria em $72 \%$ dos casos (Tabela V).

No questionário de satisfação da melhoria da deformidade houve resultados positivos em $82 \%$ dos casos (Tabela VI).

A avaliação radiológica detectou integridade protésica e óssea, com excepção de 6 fracturas de prótese (Figura 1) e 2 casos de osteólise.

Como complicações foram registadas: 4 luxações de prótese (sujeitas a revisão cirúrgica), 6 fracturas de prótese (2 revisões cirúrgicas), 2 casos de osteólise e ausência de infecções.

No questionário de satisfação global do doente, $20 \%$ consideraram o resultado cirúrgico como excelente, $7 \%$ como muito bom, $59 \%$ bom, $7 \%$ igual, $7 \%$ mau e $0 \%$ péssimo (Tabela VII). 


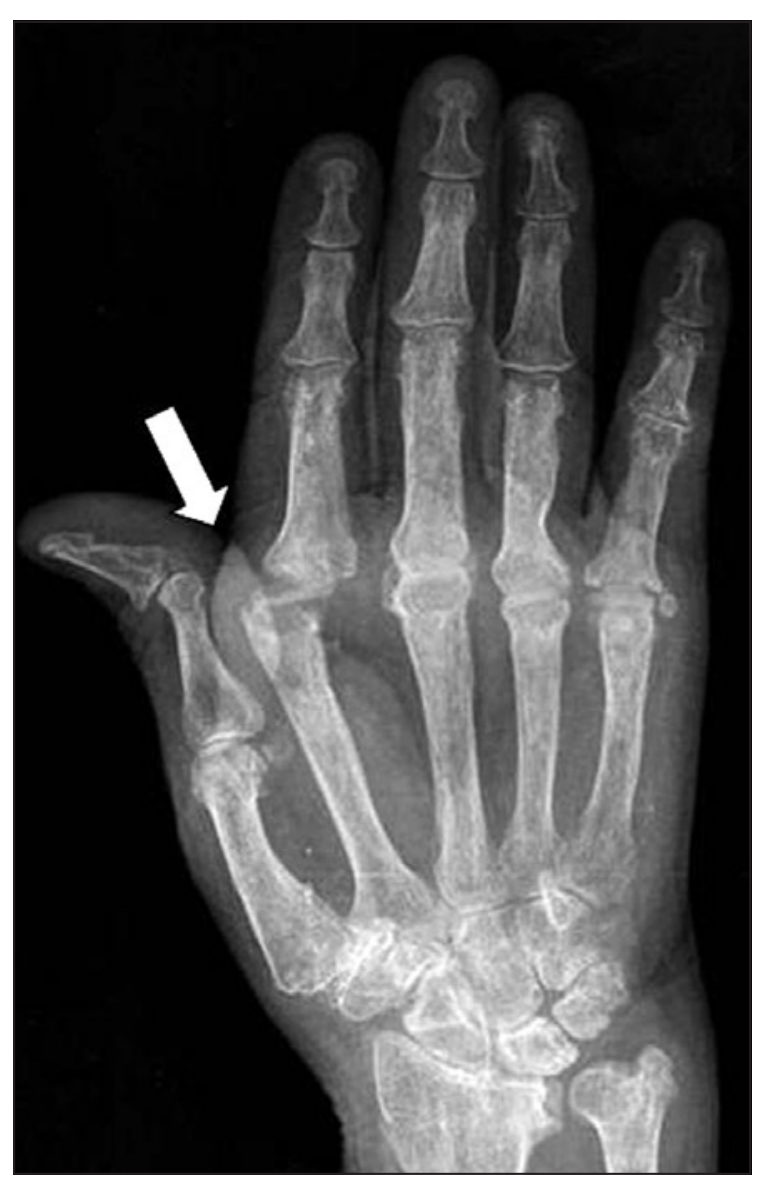

Figura 1. Fractura de prótese.

\section{DISCUSSÃO/CONCLUSÃO}

Com este trabalho de revisão ficou evidente a melhoria global, após cirurgia, da função da articulação MCF e das mãos destes doentes.

Nos vários parâmetros avaliados obtivemos resultados positivos significativos: em relação à dor registou-se $82 \%$ de resultados positivos; funcionalmente, uma melhoria objectiva de $79 \%$ dos casos e subjectivamente de $72 \%$; uma melhoria objectiva da deformidade na quase totalidade dos casos e subjectiva de $72 \%$; uma satisfação global positiva em $86 \%$ dos doentes.

Das $10 \%$ de complicações registadas, todas cursaram com boa resolução, ainda que $6 \%$ tenha necessitado de revisão cirúrgica. A luxação de prótese foi a complicação que colocou mais problemas pelo défice funcional que condiciona, obrigando a revisão cirúrgica. As fracturas de prótese normalmente não causam défices funcionais pelo que em termos clínicos não dão problemas. Provavelmente haverá um número considerável de fracturas de prótese sub-diagnosticadas, pela sua clínica frustre e pela baixa acuidade diagnóstica da radiologia convencional. A ressonância magnética nuclear seria um exame apropriado para a detecção des-
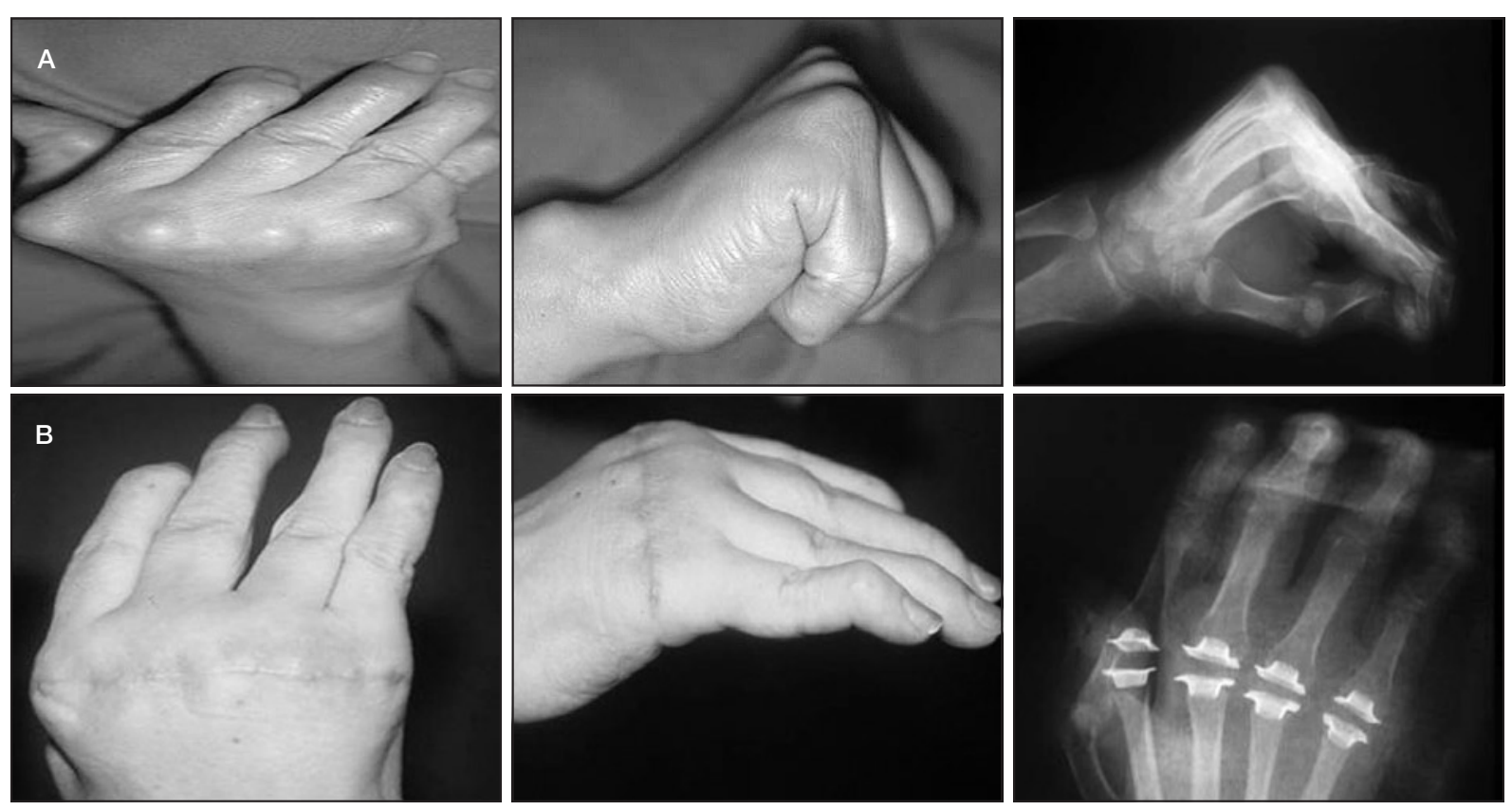

Figura 2. Comparação da deformidade da mão reumatóide com desvio cubital e volar das articulações MCF e subluxação dos tendões extensores $(A)$ com correç̧ão pós cirúrgica com prótese e correcção de tecidos moles $(B)$. 
tas fracturas mas o seu diagnóstico seria inconsequente em termos de atitude terapêutica. A percentagem de doentes insatisfeitos foi de $7 \%$, não havendo relação com nenhuma situação de complicação específica. Provavelmente, esta insatisfação terá sucedido pelas expectativas desajustadas que foram criadas por este grupo de doentes.

A radiologia ocupou um papel de destaque, a par da avaliação clínica, em todo o processo pré e pós-operatório.

Comparativamente com a literatura, os resultados desta revisão vão de encontro aos valores publicados em séries anteriores. Numa das maiores revisões publicadas em 2003 por Goldfarb e Stern $^{3}$ com revisão de 208 próteses, os parâmetros avaliados foram semelhantes nomeadamente em relação à dor, função e arco de mobilidade pós-operatório. A maior diferença centra-se nas fracturas de prótese, onde este autor refere uma percentagem de $63 \%$, contrariamente a outros trabalhos já publicados.

Como limitações do nosso estudo podemos apontar: a incapacidade de acompanhamento da alteração do arco de mobilidade no tempo, a grande complexidade da patologia reumática e dos seus doentes o que dificulta a interpretação funcional objectiva e subjectiva.

Após este conjunto de dados e avaliações concluímos que a artroplastia de interposição com prótese de silicone é uma opção eficaz e segura desde que respeitadas as indicações e planeamento cirúrgico. Desta forma sublinhamos a dor e a função como principais indicações, o «timing» adequado no tratamento cirúrgico da doença, o enquadramento global no tratamento do membro superior e a coordenação das expectativas do cirurgião com as do doente.

\section{BIBLIOGRAFIA}

1. Feldon M, Millender LH. Rheumathoid arthritis and other connective tissue diseases. En: Green DP, Hotchkiss RN, Pederson WC (Eds). Green's operative hand surgery, $5^{\text {th }}$ ed., Philadelphia: Elsevier Churchill Livingstone; 2005; 2049-136.

2. Phillip E, Wright II. Arthritic hand. En: Canale ST (Ed). Campbell's Operative Orthopaedics, 10th ed. Vol. four. Memphis: Mosby, 2003; 3689-737.

3. Goldfarb CA, Stern P. Metacarpophalangeal joint artrhoplasty. Rheumatoid arthritis, a long -term assessment; J Bone Joint Surg Am, 2003; 85: 1869-78.

4. Swanson finger joint. Performance characteristics and thirty (plus) year clinical success. Technical monograph. Toulon:
Wright Medical Technology Inc., 2005.

5. The Neuflex MCP Finger joint implant: Designed for Comfort and Performance. Warsaw: DePuy Orthopaedics, Inc., 1998.

6. Erdogan A, Weiss AP. Neuflex silastic implant in metacarpophalangeal joint arthroplasty. Orthopade. 2003; 32: 789-93. 